OPEN ACCESS

Edited by:

Izak Faiena,

Columbia University, United States

Reviewed by:

Wayne Brisbane

University of California, Los Angeles,

United States

Gopal Nand Gupta,

Loyola University Medical Center,

United States

*Correspondence:

Ugo Giovanni Falagario

ugofalagario@gmail.com

Specialty section: This article was submitted to

Genitourinary Oncology,

a section of the journal

Frontiers in Oncology

Received: 06 September 2020

Accepted: 24 November 2020

Published: 08 January 2021

Citation:

Falagario UG, Silecchia G, Bruno SM, Di Nauta M, Auciello M, Sanguedolce F, Milillo $P$, Macarini L, Selvaggio O, Carrieri G and Cormio L (2021) Does Multiparametric Magnetic

Resonance of Prostate Outperform Risk Calculators in Predicting Prostate

Cancer in Biopsy Naive Patients?

Front. Oncol. 10:603384.

doi: 10.3389/fonc.2020.603384

\section{Does Multiparametric Magnetic Resonance of Prostate Outperform Risk Calculators in Predicting Prostate Cancer in Biopsy Naïve Patients?}

Ugo Giovanni Falagario ${ }^{1 *}$, Giovanni Silecchia ${ }^{2}$, Salvatore Mariano Bruno ${ }^{1}$, Michele Di Nauta ${ }^{1}$, Mario Auciello ${ }^{1,2}$, Francesca Sanguedolce ${ }^{3}$, Paola Milillo ${ }^{4}$, Luca Macarini ${ }^{4}$, Oscar Selvaggio ${ }^{1}$, Giuseppe Carrieri ${ }^{1}$ and Luigi Cormio ${ }^{1,2}$

\footnotetext{
${ }^{1}$ Department of Urology and Organ Transplantation, University of Foggia, Foggia, Italy, ${ }^{2}$ Department of Urology, Bonomo Teaching Hospital, Andria, Italy, ${ }^{3}$ Department of Pathology, University of Foggia, Foggia, Italy, ${ }^{4}$ Department of Radiology, University of Foggia, Foggia, Italy
}

Background: European Association of Urology (EAU) guidelines recommend using riskcalculators (RCs), imaging or additional biomarkers in asymptomatic men at risk of prostate cancer $(\mathrm{PCa})$.

Objectives: To compare the performance of mpMRI, a RC we recently developed and two commonly used $\mathrm{RC}$ not including mpMRI in predicting the risk of $\mathrm{PCa}$, as well as the added value of mpMRI to each $\mathrm{RC}$.

Design, Setting, and Participants: Single-center retrospective study evaluating 221 biopsy-naïve patients who underwent prebiopsy mpMRI.

Outcome Measurements and Statistical Analysis: Patients' probabilities of any PCa and clinically significant PCa (csPC, defined as Gleason-Score $\geq 3+4$ ) were computed according to mpMRI, European Randomized Study of Screening for Prostate Cancer RC (ERSPC-RC), the Prostate Biopsy Collaborative Group RC (PBCG-RC) and the Foggia Prostate Cancer RC (FPC-RC). Logistic regression, AUC, and Decision curve analysis (DCA) were used to assess the accuracy of tested models.

Results and Limitation: The FPC-RC outperformed mpMRI in diagnosing both any PCa (AUC 0.76 vs 0.69) and csPCa (AUC 0.80 vs 0.75). Conversely mpMRI showed a higher accuracy in predicting any PCa compared to the PBCG-RC and the ERSPC-RC but similar performances in predicting csPCa. At multivariable analysis predicting csPCa and any PCa, the addition of mpMRI findings improved the accuracy of each calculator. DCA showed that the FPC-RC provided a greater net benefit than mpMRI and the other RCs. The addition of mpMRl findings improved the net benefit provided by each calculator. 


\section{Conclusions: mpMRI was outperformed by the novel FPC-RC and showed similar performances compared to the PBCG and ERSPC RCs in predicting csPCa. The addition of mpMRI findings improved the diagnostic accuracy of each of these calculators}

Keywords: prostate cancer, mpMRI, decision curve analysis, clinically significant prostate cancer, risk calculator

\section{INTRODUCTION}

In current clinical practice, the cancer detection rate (CDR) of a first extended prostate biopsy $(\mathrm{PBx})$ prompted by an elevated serum prostate-specific antigen (PSA) level and/or an abnormal digital rectal examination (DRE) is around 40\%, dropping to approximately $25 \%$ in the setting of screening programs, i.e. patients with serum PSA between 2.5 and $10 \mathrm{ng} / \mathrm{ml}(1,2)$.

To reduce the risk of unnecessary PBxs, current European Association of Urology (EAU) guidelines (3) provide a strong recommendation to offer further risk-assessment to asymptomatic men with normal DRE but PSA levels between 2 and $10 \mathrm{ng} / \mathrm{ml}$ prior to performing PBx. Such "further risk assessment" should be done by one of following tools: i) risk-calculator (RC); ii) imaging; iii) an additional serum or urine-based test (3). Interestingly, while this recommendation has remained unchanged in 2018 and 2019 Guidelines, the 2020 Guidelines provide a weak recommendation to perform mpMRI in any patient with clinical suspicion for prostate cancer (PCa). If mpMRI demonstrates lesion(s) suspicious for $\mathrm{PCa}$, systematic and target biopsy should be performed, whereas biopsy can be avoided when mpMRI is negative and the clinical suspicion of PCa is low. By doing so, the 2020 Guidelines somehow bind the decision to perform mpMRI to the clinical suspicion of PCa which is well determined by available RCs.

RCs are designed to determine the risk of an individual harboring PCa by entering into a statistical model his clinical parameters. To date, several calculators have been developed and externally validated; a few also include mpMRI findings and biomarkers but questions remain on the additional value provided by such tests. Indeed, a recent study aiming at comparing and externally validating prostate cancer RCs incorporating mpMRI demonstrated that the addition of mpMRI parameters to RCs based on standard clinical variables was limited (3). Overall, available information regarding the use of RCs, mpMRI or biomarkers as triage test and the utility of combining them remain scarce.

The present study therefore aimed to compare the performance of mpMRI with the performances of two commonly-used externally-validated calculators not including $\operatorname{mpMRI}(4,5)$ and a novel externally-validated calculator we recently developed (6) in predicting the risk of harboring $\mathrm{PCa}$, as well as the added value of mpMRI to each RC.

\section{PATIENTS AND METHODS}

Our Internal Review Board which approved the database on prostate biopsy was queried to identify patients who underwent
mpMRI and trans-rectal prostate biopsy at our institution under the clinical suspicion of PCa. The patient population used for the development of our RC was not included in the present study.

Prostate mpMRI was triggered by PSA higher than $3.0 \mathrm{ng} / \mathrm{ml}$ and/or abnormal DRE and were interpreted by a single dedicated radiologist (PM) with 10 years of experience in prostate MRI, using the PIRADSv2.0 recommendations (7).

All patients underwent PSA measurement before DRE and transrectal ultrasound (TRUS). Uroflowmetry (UFM) was carried out before $\mathrm{PBx}$, waiting for the patient to report a strong sensation to void.

MRI examinations were performed using a 1.5 T MR scanner (Achieva, Philips Healthcare, Best, The Netherlands) and surface array coils (SENSE Flex surface) or with endorectal coil (ERC) combined with 16-channel surface coil (TORSO-XL coil). The mpMRI protocol was compliant with PIRADs 2.0 recommendations (7) and consisted of: A. Turbo-Spin-Echo (TSE) T2-weighed imaging in axial, coronal and sagittal planes [repetition time (TR) 5,300, echo time (TE) $150 \mathrm{~ms}$, slice thickness $3 \mathrm{~mm}$, field of view (FOV) $180 \times 180$, number of signal averaged (NSA) 8]; B. TSE T1-weighed imaging in axial plane [TR/TE 400-650/12 ms, thickness $3 \mathrm{~mm}$, FOV $180 \times 180$, NSA 3]; C. Diffusion-weighted imaging sequence (DWI) in the axial plane [TR/TE $3,481 / 92 \mathrm{~ms}$, slice thickness $3 \mathrm{~mm}$, FOV $180 \times 220$, NSA 4, b-values $0-500$ $\left.1000-1,500 / 2,000 \mathrm{~s} / \mathrm{mm}^{2}\right]$; D. Dynamic contrast enhanced prostate MRI was performed using a T1-weighted high resolution isotropic volume examination (THRIVE) on the axial plane [TR/TE 4.5/2.2 ms, slice thickness $3 \mathrm{~mm}$, FOV $184 \times 220$, NSA 1] following injection of $0.1 \mathrm{ml} / \mathrm{kg}$ of gadobutrol followed by $20 \mathrm{ml}$ of saline solution using an automatic injector at a rate of $2 \mathrm{ml} / \mathrm{s}$.

In accordance with the current EAU guidelines, patients with negative mPMRI (PIRADS 1 and 2 lesions were considered to be negative) received a standard ultrasound guided transrectal PBx using our 18-core template (8); those with a positive mpMRI received a transrectal electromagnetic-tracked MRI/US fusion guided biopsy (Navigo, UC-CARE, Yokneam, ISR). To avoid large differences in the number of cores, we attempted to include the two target cores from each mpMRI-suspicious lesions into our 18-core biopsy scheme (SBx). All procedures were carried out by two of us (OS, GS) under local non-infiltrative anesthesia $(8,9)$.

A single, dedicated uropathologist (FS) reviewed all biopsy specimens according to International Society of Urological Pathology; Gleason Grade Groups (GGG) were assigned to each patient (10). Contemporary diagnostic criteria for highgrade prostatic intraepithelial neoplasia (HGPIN), atypical small acinar proliferation (ASAP) of prostate (11), and PCa were followed. 


\section{Statistical Analysis}

Outcomes of the present study were probabilities of any PCa $(G G G \geq 1)$ and clinically significant PCa (csPC defined as $\mathrm{GGG} \geq 2$ ) as assessed by mpMRI alone, or by one RC alone, namely the European Randomized Study of Screening for Prostate Cancer (ERSPC) (4), the Prostate Biopsy Collaborative Group (PBCG) (5) and the Foggia Prostate Cancer (FPC) RCs (6), or by adding mpMRI to each RC.

Our primary objective was to compare the accuracy of mpMRI and the FPC-RC. As a secondary objective we sought to compare the accuracy of mpMRI with two of the most used available RCs. Finally, we determined the added value of mpMRI to each of the tested models.

For descriptive statistics, continuous variables were reported as medians and interquartile ranges, whereas categorical variables were reported as rates. Patients' probabilities of any $\mathrm{PCa}$ as well as csPCa were computed applying the coefficients (available upon request to the authors of the original publications) to the logit functions for the ERSPC-RC and the FPC-RC. Conversely, individual probability of i. No cancer, ii. Low grade cancer, and iii. High grade cancer for the PBCG-RC was computed using the coefficients and formulas provided by the authors as supplementary materials (5).

Univariable logistic regression was carried out in order to compare each RC against mpMRI as predictors of the outcomes of interest.

Three models predicting any $\mathrm{PCa}$ and three models predicting csPCa were created adding mpMRI to the individual risk computed for each calculator, in a multivariable model. Since the PBCG-RC was developed using multinomial regression (i.e. it provides risk of no cancer, low-grade cancer, high-grade cancer), the risk prediction for any cancer was computed as 1-risk of no cancer and was identical to the risk of low-grade cancer + risk of high-grade cancer.

The corresponding area under receiver operating characteristic (ROC) curve (AUC) and decision curve analysis (DCA) were used to assess the predictive accuracy and clinical benefit of tested models.

Statistical analyses were performed according to the latest guidelines (12) using STATA 15 (StataCorp LP, College Station, TX, USA). Significance was set at $\alpha=0.05$.

\section{RESULTS}

Between January 2017 and October 2019, a total of 415 patients underwent mpMRI and PBx at our Institution. Men receiving five alfa-reductase inhibitors $(\mathrm{N}=50)$, or who had previously undergone $\mathrm{PBx}(\mathrm{N}=174)$, or invasive treatment for benign prostatic hyperplasia $(n=11)$, or with dwelling urethral catheters $(\mathrm{N}=5)$, or with a voided volume of less than $150 \mathrm{ml}(\mathrm{N}=7)$ were excluded from the present study. Patients with PSA $>20 \mathrm{ng} / \mathrm{ml}$ $(\mathrm{N}=18)$ were also excluded as we found them to have a too high risk $(>75 \%)$ of harboring PCa.

After the exclusion criteria, the final population included 221 biopsy naïve patients with complete data. Patients characteristics are summarized in Table 1; 43 patients (19.5\%) had a negative mpMRI (PIRADS 1-2), thus underwent 18-core US guided transrectal $\mathrm{PBx}$, whereas the remaining 168 underwent mpMRI/US guided fusion PBx. Their PIRADS score was 3, 4, and 5 in 35 (15.8\%), 120 (54.3\%) and 23 (10.4\%), respectively.

Any PCa and csPCa detection rates were $53.8 \%(\mathrm{n}=119)$ and $27.1 \%(n=60)$, respectively. The negative predictive value of mpMRI (PIRADS 1-2) in predicting any PCa and csPCa was 76.7 and $97.7 \%$ respectively. The positive predictive value of mpMRI (PIRADS 3-4-5) was $61.2 \%$ for any PCa and $33.1 \%$ for csPCa.

Univariable analysis predicting the outcomes of interest is shown in Table 2. RCs and mpMRI PIRADS score were all significant predictors of any PCa and csPCa $(\mathrm{p}<0.05)$.

The FPC-RC outperformed mpMRI in diagnosing both any PCa (AUC 0.76 vs 0.69) and csPCa (AUC 0.80 vs 0.75). Conversely mpMRI showed a higher accuracy in predicting any PCa compared to the PBCG-RC and the ERSPC-RC but similar performances in predicting csPCa (Table 2, Figures 1A-C).

Multivariable analysis showed that the addition of mpMRI findings improved the diagnostic accuracy of each calculator in predicting both csPCa and any PCa. The model derived from the addition of mpMRI to the FPC-Rc showed the highest accuracy in diagnosing both any PCa (AUC 0.78) and csPCa (AUC 0.87) (Table 3, Figures 1B, D).

Finally, DCA showed that the FPC-RC provided greater net benefit than mpMRI in predicting any PCa and csPCa. Conversely mpMRI had a higher net benefit compared to the other RCs. Again, the addition of mpMRI findings improved the net benefit provided by each calculator benefit (Figure 2).

\section{DISCUSSION}

Over the last years, mpMRI has gained popularity as a reliable tool in localizing specific regions of the prostate highly suspicious

TABLE 1 | Descriptive characteristics of the study population.

\begin{tabular}{lc}
\hline & $\begin{array}{c}\text { Overall population } \\
\mathbf{N}=\mathbf{2 2 1}\end{array}$ \\
\hline Age & $66.0(60.0,71.0)$ \\
DRE, $\mathbf{n}$ (\%) & \\
Negative & $123(55.7 \%)$ \\
Suspicious & $98(44.3 \%)$ \\
Family History & \\
Negative & $108(76.1 \%)$ \\
Positive & $34(23.9 \%)$ \\
PSA, ng/mI & $5.5(4.1,7.4)$ \\
PSA density & $0.11(0.07,0.15)$ \\
Prostate volume, cc & $52.0(39.0,69.0)$ \\
PIRADS & \\
1-2 & $43(19.5 \%)$ \\
3 & $35(15.8 \%)$ \\
4 & $120(54.3 \%)$ \\
5 & $23(10.4 \%)$ \\
Any Cancer & $119(53.8 \%)$ \\
Cs Cancer & $60(27.1 \%)$ \\
NPV of PIRADS 1-2 & $97.8 \%(42 / 43)$ \\
PPV of PIRADS 3-4-5 & $33.1 \%(59 / 178)$
\end{tabular}


TABLE 2 | Univariable analysis predicting any cancer and csPCa using risk calculators and mpMRI.

\begin{tabular}{|c|c|c|c|c|c|c|c|c|}
\hline & \multicolumn{4}{|c|}{ OUTCOME: ANY PCa } & \multicolumn{3}{|c|}{ OUTCOME: csPCa } & AUC \\
\hline FPC-RC, per unit & 1.05 & $1.03,1.07$ & $<0.001$ & 0.760 & 1.06 & $1.04,1.08$ & $<0.001$ & 0.801 \\
\hline ERSPC-RC, per unit & 1.00 & $1.00,1.00$ & 0.001 & 0.614 & 1.09 & $1.06,1.13$ & $<0.001$ & 0.749 \\
\hline \multicolumn{9}{|l|}{ MRI highest PIRADS } \\
\hline $1-2$ & Ref. & & & 0.690 & Ref. & & & 0.754 \\
\hline 5 & 11.88 & $3.52,40.14$ & $<0.001$ & & 65.33 & $7.59,562.40$ & $<0.001$ & \\
\hline
\end{tabular}
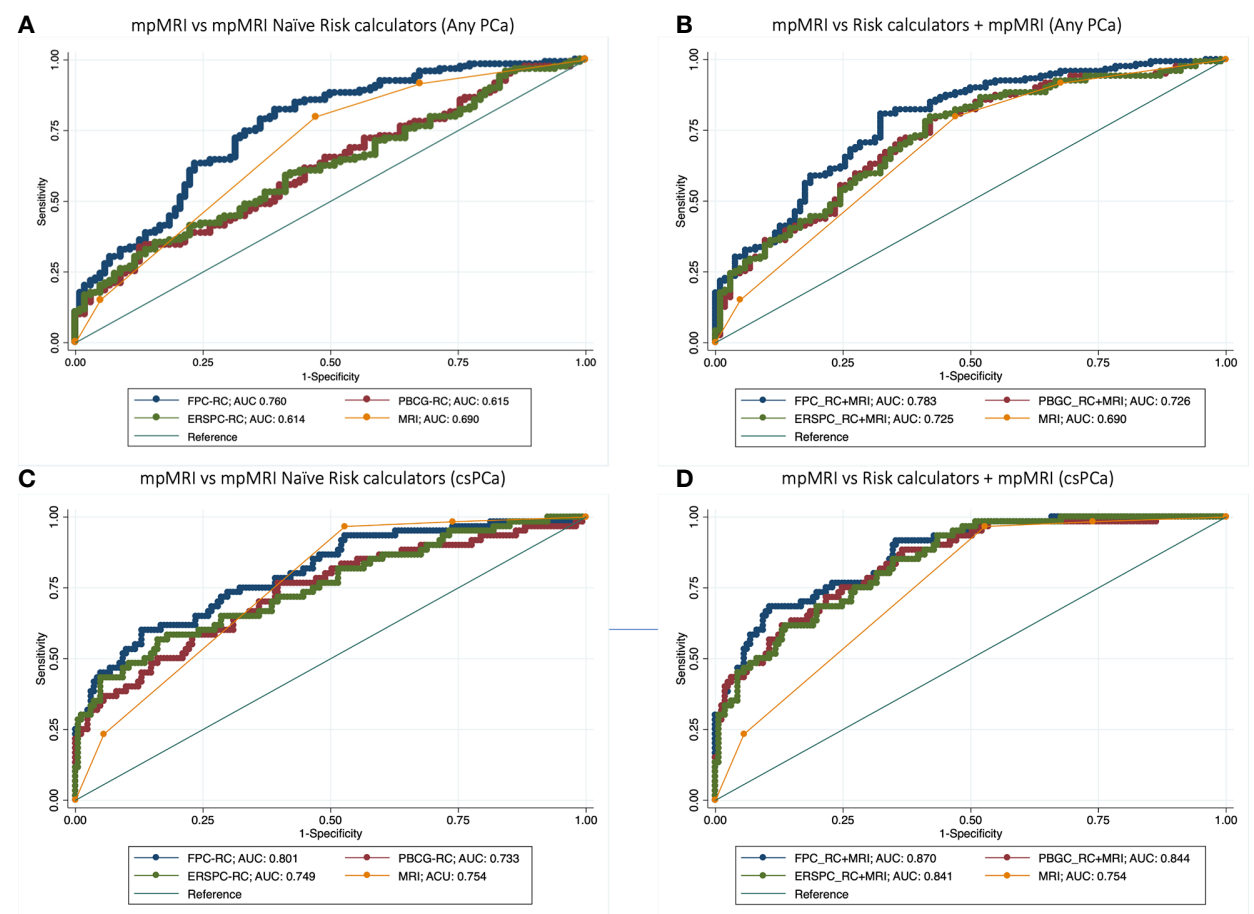

FIGURE 1 | Receiver operator curve (ROC) analysis comparing accuracy of mpMRI vs mpMRI naiive Risk calculators (RCs) (A-C), and model based on mpMRI + RCs (B-D) for detecting any PCa (A, B) and clinically significant prostate cancer

(C, D).

for csPCa; therefore, there is a trend to recommend it as the most efficient tool in predicting $\mathrm{PCa}$ at $\mathrm{PBx}$ (13). Such recommendation is however based on prospective studies in high volume tertiary cancer centers that do not reflect everyday practice in less experienced centers (14-16). Indeed, mpMRI suffers a great inter-reader and inter-center variability $(13,17,18)$; moreover, it is expensive. and not all institutions may afford to test every patient at risk for PCa. Conversely, RCs are freely available online and have been proved to be effective in several external validation cohorts.

The first interesting finding of our study was that the FPC$\mathrm{RC}$, which has recently been externally validated in a cohort of 1,377 biopsy naïve patients from 11 institutions (19), outperformed mpMRI in predicting PBx outcomes. This finding somehow further supports the clinical value of benign prostatic obstruction parameters in the evaluation of patients with PCa suspicion
(20-22). Differently from mpMRI, the FPC-RC is a freely available and almost inexpensive tool that can be easily used during any medical consultation. Should our findings be replicated in further external cohorts, the FPC-RC may become an essential tool for patients requiring "further risk assessment" prior to performing $\mathrm{PBx}$.

When compared to other RCs, mpMRI outperformed the ERSPC-RC and the PBCG-RC in predicting any PCa but showed similar performances in predicting csPCa.

Our study also aimed to answer the relevant question whether combining diagnostic tools may improve their diagnostic accuracy. Overall, the addition of mpMRI findings improved the diagnostic accuracy of each calculator in predicting both csPCa and any PCa. Our findings are in line with those from a single center study whereby the diagnostic accuracy of 4 RCs incorporating mpMRI 
TABLE 3 | Multivariable analysis predicting any PCa and csPCa.

\begin{tabular}{|c|c|c|c|c|c|c|c|c|}
\hline & \multicolumn{4}{|c|}{ OUTCOME: ANY PCa } & \multicolumn{4}{|c|}{ OUTCOME: csPCa } \\
\hline \multicolumn{9}{|l|}{ MODEL-1 } \\
\hline $1-2$ & Ref. & & & & Ref. & & & \\
\hline 3 & 1.91 & $0.67,5.47$ & 0.226 & & 1.67 & $0.08,33.59$ & 0.738 & \\
\hline \multicolumn{9}{|l|}{ MODEL-2 } \\
\hline \multicolumn{8}{|l|}{ MRI highest Pirads } & 0.844 \\
\hline $1-2$ & Ref. & & & & Ref. & & & \\
\hline 3 & 2.28 & $0.85,6.14$ & 0.104 & & 1.30 & $0.08,22.33$ & 0.855 & \\
\hline 4 & 5.59 & $2.49,12.55$ & $<0.001$ & & 23.75 & $3.06,184.34$ & 0.002 & \\
\hline $1-2$ & Ref. & & & & Ref. & & & \\
\hline 3 & 2.29 & $0.85,6.17$ & 0.102 & & 1.66 & $0.09,30.08$ & 0.731 & \\
\hline 4 & 5.58 & $2.48,12.54$ & $<0.001$ & & 24.23 & $2.87,204.38$ & 0.003 & \\
\hline 5 & 9.66 & $2.80,33.28$ & $<0.001$ & & 45.37 & $4.62,445.97$ & 0.001 & \\
\hline
\end{tabular}

Three models were created adding mpMRl to each risk calculator.
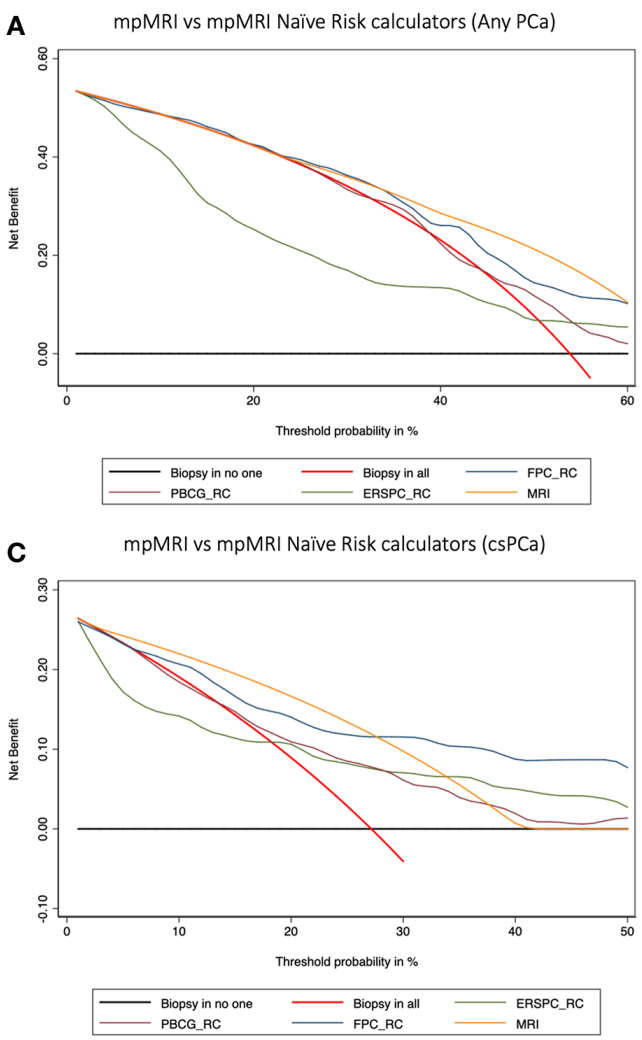

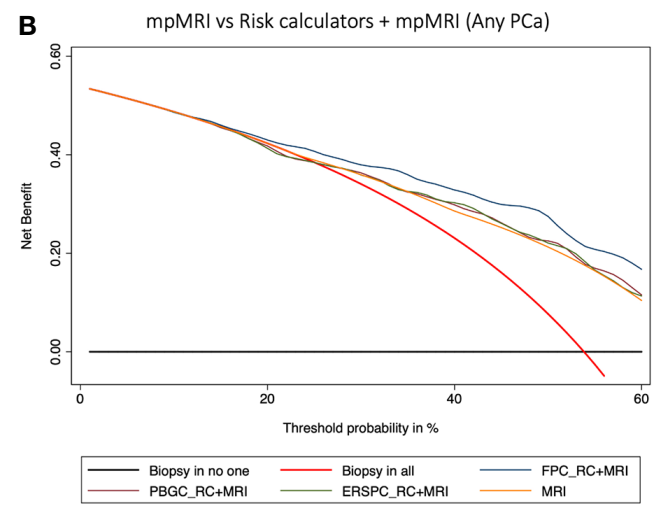

D

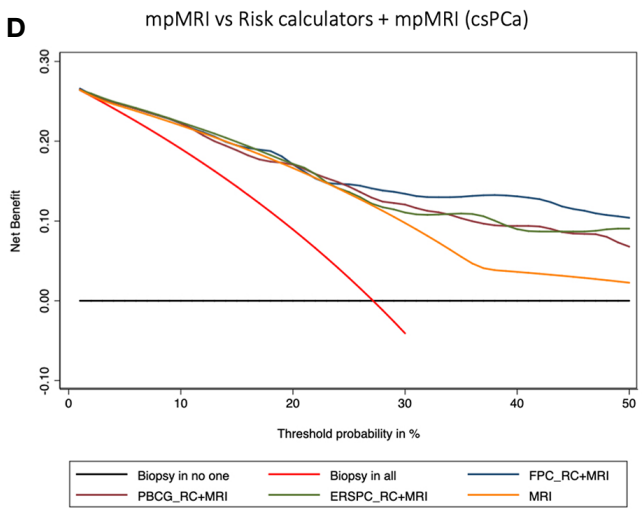

FIGURE 2 | Decision curve analysis (DCA) comparing the clinical utility of mpMRI vs mpMRI naiive risk calculators (RCs) (A-C), and model-based on mpMRI + RCs (B-D) for detecting any PCa (A, B) and clinically significant prostate cancer (C, D). The DCA simulates two scenarios: one in which all patients would receive biopsy (biopsy in all) and one in which none undergoes biopsy (biopsy in no one). Clinically useful models lie above these scenarios. Models including mpMRI +RCs showed a higher net benefit at each threshold probability and thus outperformed mpMRI alone in determining the need for a prostate biopsy. 
(23-26) was compared with that of the ERSPC-RC (4) and PBCG-RC (5) in a population of 468 patients. The four RCs incorporating mpMRI parameters provided better discrimination, calibration, and clinical usefulness; however, none of the six calculators demonstrated clinical benefit against a "biopsy all" strategy at thresholds of less than 15\% (27). This finding underlines a potentially relevant limitation of RCs; specifically, a model that shows benefit at high thresholds of probability is clinically useless in a screening setting since the decision to perform a biopsy is especially difficult in patients with borderline risk.

In the present study, DCA showed that the combination of mpMRI and RCs provided a greater benefit than the "biopsy all" strategy at low thresholds. Having said this, additional external validation studies in different biopsy settings are warranted since the clinical utility of these models could be cohort dependent. It is also worth mentioning that calculators including mpMRI, though outperforming the mpMRI naïve ones, involve obtaining mpMRI in all patients and this may not be afforded in centers with limited resources (28). Conversely, mpMRI naïve RCs offer the unique opportunity to potentially tailor further testing, such as mpMRI and PBx itself, on an individual basis. Indeed, it has been pointed out that RCs and biomarkers may help in selecting patients who could benefit from mpMRI and PBx and patients with a very low risk of csPCa in whom the positive predictive value of mpMRI is low and mpMRI and PBx should be avoided (29-31).

The findings of this study have to be seen in light of some limitations. First, the FPC-RC was developed at our institution, and this can explain its better performance compared to the other tested RCs. Even if the patient population used for the development of the RC was not included in the present study, this cannot be considered an external validation study. Other potential study limitations include its relatively small sample size and its retrospective nature; however, we elected to use strict inclusion criteria and data were prospectively collected. Finally, we did not test novel and promising tools such as bi-parametric MRI (32) and novel biomarkers (33), but this would have been beyond the aim of a study comparing currently available tests.

\section{REFERENCES}

1. Louie KS, Seigneurin A, Cathcart P, Sasieni P. Do prostate cancer risk models improve the predictive accuracy of PSA screening? A meta-analysis. Ann Oncol (2015) 26(5):848-64. doi: 10.1093/annonc/mdu525

2. Lughezzani G, Lazzeri M, Larcher A, Lista G, Scattoni V, Cestari A, et al. Development and internal validation of a Prostate Health Index based nomogram for predicting prostate cancer at extended biopsy. J Urol (2012) 188(4):1144-50. doi: 10.1016/j.juro.2012.06.025

3. Mottet N, van den Bergh RCN, Briers E, Cornford P, De Santis M, Fanti S, et al. EAU - ESTRO - ESUR - SIOG Guidelines on Prostate Cancer 2020. In: . European Association of Urology Guidelines. 2020 Edition. Arnhem, The Netherlands: European Association of Urology Guidelines Office (2020).

4. Roobol MJ, Verbeek JFM, van der Kwast T, Kummerlin IP, Kweldam CF, van Leenders G. Improving the Rotterdam European Randomized Study of Screening for Prostate Cancer Risk Calculator for Initial Prostate Biopsy by Incorporating the 2014 International Society of Urological Pathology Gleason Grading and Cribriform growth. Eur Urol (2017) 72(1):45-51. doi: 10.1016/j.eururo.2017.01.033

5. Ankerst DP, Straubinger J, Selig K, Guerrios L, De Hoedt A, Hernandez J, et al. A Contemporary Prostate Biopsy Risk Calculator Based on Multiple Heterogeneous Cohorts. Eur Urol (2018) 74(2):197-203. doi: 10.1016/ j.eururo.2018.05.003

\section{CONCLUSIONS}

The present study pointed out that mpMRI was outperformed by the novel FPC-RC and showed similar performances compared to the PBCG and ERSPC risk calculators in predicting csPCa. The addition of mpMRI findings improved the diagnostic accuracy of each of these calculators. Further studies are needed to assess how these findings can be used to safely avoid unnecessary biopsies.

\section{DATA AVAILABILITY STATEMENT}

The raw data supporting the conclusions of this article will be made available by the authors, without undue reservation.

\section{ETHICS STATEMENT}

The studies involving human participants were reviewed and approved by the University of Foggia Ethics committee. The patients/participants provided their written informed consent to participate in this study.

\section{AUTHOR CONTRIBUTIONS}

LC, UF, GC, LM, and OS conceived and designed the study. GS, MB, MN, MA, and PM acquired the data. LC, UF, and PM analyzed and interpreted the data. LC, UF, GS, and MB drafted the manuscript. LC, GC, OS, and LM made critical revisions of the manuscript for important intellectual content. LC and UF conducted the statistical analysis. LC, GC, OS, and LM supervised the study. All authors contributed to the article and approved the submitted version.

6. Cormio L, Cindolo L, Troiano F, Marchioni M, Di Fino G, Mancini V, et al. Development and Internal Validation of Novel Nomograms Based on Benign Prostatic Obstruction-Related Parameters to Predict the Risk of Prostate Cancer at First Prostate Biopsy. Front Oncol (2018) 8:438:438. doi: 10.3389/ fonc. 2018.00438

7. Muller BG, Shih JH, Sankineni S, Marko J, Rais-Bahrami S, George AK, et al. Prostate Cancer: Interobserver Agreement and Accuracy with the Revised Prostate Imaging Reporting and Data System at Multiparametric MR Imaging. Radiology (2015) 277(3):741-50. doi: 10.1148/radiol.2015142818

8. Cormio L, Lorusso F, Selvaggio O, Perrone A, Sanguedolce F, Pagliarulo V, et al. Noninfiltrative anesthesia for transrectal prostate biopsy: a randomized prospective study comparing lidocaine-prilocaine cream and lidocaineketorolac gel. Urol Oncol (2013) 31(1):68-73. doi: 10.1016/j.urolonc. 2010.09.004

9. Cormio L, Pagliarulo V, Lorusso F, Selvaggio O, Perrone A, Sanguedolce F, et al. Combined perianal-intrarectal (PI) lidocaine-prilocaine (LP) cream and lidocaine-ketorolac gel provide better pain relief than combined PI LP cream and periprostatic nerve block during transrectal prostate biopsy. BJU Int (2012) 109(12):1776-80. doi: 10.1111/j.1464-410X.2011.10622.x

10. Epstein JII, Egevad L, Amin MB, Delahunt B, Srigley JR, Humphrey PA, et al. The 2014 International Society of Urological Pathology (ISUP) Consensus Conference on Gleason Grading of Prostatic Carcinoma: Definition of 
Grading Patterns and Proposal for a New Grading System. Am J Surg Pathol (2016) 40(2):244-52. doi: 10.1097/PAS.0000000000000530

11. Sanguedolce F, Cormio A, Musci G, Troiano F, Carrieri G, Bufo P, et al. Typing the atypical: Diagnostic issues and predictive markers in suspicious prostate lesions. Crit Rev Clin Lab Sci (2017) 54(5):309-25. doi: 10.1080/ 10408363.2017.1363155

12. Assel M, Sjoberg D, Elders A, Wang X, Huo D, Botchway A, et al. Guidelines for reporting of statistics for clinical research in urology. BJU Int (2019) 123 (3):401-10. doi: 10.1111/bju.14640

13. Wajswol E, Winoker IS, Anastos H, Falagario U, Okhawere K, Martini A, et al. A cohort of transperineal electromagnetically tracked magnetic resonance imaging/ultrasonography fusion-guided biopsy: assessing the impact of interreader variability on cancer detection. BJU Int (2019) 125(4):531-40. doi: 10.1111/bju.14957

14. Ahmed HU, El-Shater Bosaily A, Brown LC, Gabe R, Kaplan R, Parmar MK, et al. Diagnostic accuracy of multi-parametric MRI and TRUS biopsy in prostate cancer (PROMIS): a paired validating confirmatory study. Lancet (2017) 389(10071):815-22. doi: 10.1016/S0140-6736(16)32401-1

15. Kasivisvanathan V, Rannikko AS, Borghi M, Panebianco V, Mynderse LA, Vaarala MH, et al. MRI-Targeted or Standard Biopsy for Prostate-Cancer Diagnosis. N Engl J Med (2018) 378(19):1767-77. doi: 10.1056/NEJMoa1801993

16. Rouviere O, Puech P, Renard-Penna R, Claudon M, Roy C, Mege-Lechevallier F, et al. Use of prostate systematic and targeted biopsy on the basis of multiparametric MRI in biopsy-naive patients (MRI-FIRST): a prospective, multicentre, paired diagnostic study. Lancet Oncol (2019) 20(1):100-9. doi: 10.1016/S1470-2045(18)30569-2

17. Rosenkrantz AB, Ginocchio LA, Cornfeld D, Froemming AT, Gupta RT, Turkbey B, et al. Interobserver Reproducibility of the PI-RADS Version 2 Lexicon: A Multicenter Study of Six Experienced Prostate Radiologists. Radiology (2016) 280(3):793-804. doi: 10.1148/radiol.2016152542

18. Smith CP, Harmon SA, Barrett T, Bittencourt LK, Law YM, Shebel H, et al. Intra- and interreader reproducibility of PI-RADSv2: A multireader study. J Magn Reson Imaging (2019) 49(6):1694-703. doi: 10.1002/jmri.26555

19. Cindolo L, Bertolo R, Minervini A, Sessa F, Muto G, Bove P, et al. External validation of Cormio nomogram for predicting all prostate cancers and clinically significant prostate cancers. World J Urol (2020) 38(10):2555-61. doi: 10.1007/s00345-019-03058-1

20. Cormio L, Lucarelli G, Netti GS, Stallone G, Selvaggio O, Troiano F, et al. Post-void residual urinary volume is an independent predictor of biopsy results in men at risk for prostate cancer. Anticancer Res (2015) 35(4):2175-82.

21. Cormio L, Lucarelli G, Selvaggio O, Di Fino G, Mancini V, Massenio P, et al. Absence of Bladder Outlet Obstruction Is an Independent Risk Factor for Prostate Cancer in Men Undergoing Prostate Biopsy. Medicine (Baltimore) (2016) 95(7):e2551. doi: 10.1097/MD.0000000000002551

22. Cicione A, Cormio L, Cantiello F, Palumbo IM, De Nunzio D, Lima E, et al. Presence and severity of lower urinary tract symptoms are inversely correlated with the risk of prostate cancer on prostate biopsy. Minerva Urol Nefrol (2017) 69(5):486-92. doi: 10.23736/S0393-2249.17.02737-0

23. Radtke JP, Wiesenfarth M, Kesch C, Freitag MT, Alt CD, Celik K, et al. Combined Clinical Parameters and Multiparametric Magnetic Resonance Imaging for Advanced Risk Modeling of Prostate Cancer-Patient-tailored Risk Stratification Can Reduce Unnecessary Biopsies. Eur Urol (2017) 72 (6):888-96. doi: 10.1016/j.eururo.2017.03.039

24. van Leeuwen PJ, Hayen A, Thompson JE, Moses D, Shnier R, Bohm M, et al. A multiparametric magnetic resonance imaging-based risk model to determine the risk of significant prostate cancer prior to biopsy. BJU Int (2017) 120 (6):774-81. doi: 10.1111/bju.13814
25. Mehralivand S, Shih JH, Rais-Bahrami S, Oto A, Bednarova S, Nix JW, et al. A Magnetic Resonance Imaging-Based Prediction Model for Prostate Biopsy Risk Stratification. JAMA Oncol (2018) 4(5):678-85. doi: 10.1001/ jamaoncol.2017.5667

26. Alberts AR, Roobol MJ, Verbeek JFM, Schoots IG, Chiu PK, Osses DF, et al. Prediction of High-grade Prostate Cancer Following Multiparametric Magnetic Resonance Imaging: Improving the Rotterdam European Randomized Study of Screening for Prostate Cancer Risk Calculators. Eur Urol (2019) 75(2):310-8. doi: 10.1016/j.eururo.2018.07.031

27. Saba K, Wettstein MS, Lieger L, Hotker AM, Donati OF, Moch H, et al. External Validation and Comparison of Prostate Cancer Risk Calculators Incorporating Multiparametric Magnetic Resonance Imaging for Prediction of Clinically Significant Prostate Cancer. J Urol (2019) 203(4):719-26. doi: 10.1097/JU.0000000000000622. 101097JU0000000000000622.

28. Perez IM, Jambor I, Kauko T, Verho J, Ettala O, Falagario U, et al. Qualitative and Quantitative Reporting of a Unique Biparametric MRI: Towards Biparametric MRI-Based Nomograms for Prediction of Prostate Biopsy Outcome in Men With a Clinical Suspicion of Prostate Cancer (IMPROD and MULTI-IMPROD Trials). J Magn Reson Imaging (2020) 51(5):1556-67. doi: $10.1002 /$ jmri.26975

29. Falagario UG, Martini A, Wajswol E, Treacy PJ, Ratnani P, Jambor I, et al. Avoiding Unnecessary Magnetic Resonance Imaging (MRI) and Biopsies: Negative and Positive Predictive Value of MRI According to Prostate-specific Antigen Density, 4Kscore and Risk Calculators. Eur Urol Oncol (2019) 3 (5):700-4. doi: 10.1016/j.euo.2019.08.015

30. Beksac AT, Cumarasamy S, Falagario U, Xu P, Takhar M, Alshalalfa M, et al. Multiparametric Magnetic Resonance Imaging Features Identify Aggressive Prostate Cancer at the Phenotypic and Transcriptomic Level. J Urol (2018) 200(6):1241-9. doi: 10.1016/j.juro.2018.06.041

31. Falagario UG, Jambor I, Lantz A, Ettala O, Stabile A, Taimen P, et al. Combined Use of Prostate-specific Antigen Density and Magnetic Resonance Imaging for Prostate Biopsy Decision Planning: A Retrospective Multi-institutional Study Using the Prostate Magnetic Resonance Imaging Outcome Database (PROMOD). Eur Urol Oncol (2020). doi: 10.1016/ j.euo.2020.08.014

32. Perez IM, Jambor I, Kauko T, Verho J, Ettala O, Falagario U, et al. Qualitative and Quantitative Reporting of a Unique Biparametric MRI: Towards Biparametric MRI-Based Nomograms for Prediction of Prostate Biopsy Outcome in Men With a Clinical Suspicion of Prostate Cancer (IMPROD and MULTI-IMPROD Trials). J Magn Reson Imaging (2019) 51(5):1556-67. doi: $10.1002 /$ jmri.26975

33. Stallone G, Cormio L, Netti GS, Infante B, Selvaggio O, Fino GD, et al. Pentraxin 3: a novel biomarker for predicting progression from prostatic inflammation to prostate cancer. Cancer Res (2014) 74(16):4230-8. doi: 10.1158/0008-5472.CAN-14-0369

Conflict of Interest: The authors declare that the research was conducted in the absence of any commercial or financial relationships that could be construed as a potential conflict of interest.

Copyright (C) 2021 Falagario, Silecchia, Bruno, Di Nauta, Auciello, Sanguedolce, Milillo, Macarini, Selvaggio, Carrieri and Cormio. This is an open-access article distributed under the terms of the Creative Commons Attribution License (CC BY). The use, distribution or reproduction in other forums is permitted, provided the original author(s) and the copyright owner(s) are credited and that the original publication in this journal is cited, in accordance with accepted academic practice. No use, distribution or reproduction is permitted which does not comply with these terms. 